\title{
Instruction effects on size and distance judgments
}

\author{
ZITA E. TYER, JOHN A. ALLEN, and ROBERT PASNAK \\ George Mason University, Fairfax, Virginia
}

\begin{abstract}
The hypotheses that size judgments of unfamiliar objects are affected by distance information, and distance judgments by size information, were tested. Subjects made size or distance estimates in a cue-reduced situation, with or without distance or size information, and also made calibrated estimates in full-cue conditions. Size judgments in the no-information condition were correlated with the retinal image, whereas distance information produced size estimates closer to the actual size of the objects. Subjects given no information about size produced distance estimates that were randomly distributed, whereas size information yielded a weak effect in the appropriate direction. Implications for the size-distance invariance hypothesis and the specific distance tendency are discussed.
\end{abstract}

No previous investigation has assessed both the effects of size information on distance judgments and the effect of distance information on size judgments for varying retinal images. The purpose of the present study was to investigate these variables in a single experimental setting in which cue reduction was assured.

The majority of the studies in this area focus on size or distance judgments under familiar size instructions or with off-sized familiar objects. Several studies (Fitzpatrick, Pasnak, \& Tyer, 1982; Gogel, 1968; Gogel \& Mertens, 1967) have shown that the judged size of a familiar object corresponds very well to its real size, and judged distance corresponds to the actual distance at which that size would produce the given retinal size. In other studies (Baird, 1963; Park \& Michaelson, 1974), an object is presented and the observer is told that it is the same size as a familiar object; distance judgments correspond quite well to the distance at which that object would produce the given retinal image.

It is not clear what the effect would be if an instruction regarding an object's size was not associated with a familiar object. In this case, an observer would have to interpret the object in terms of the retinal image and cognitive information. Coltheart (1970) reported that subjects could do this very well; Park and Michaelson (1974), on the other hand, reported that size instruction had no effect on distance judgments. Since it is not possible to determine why these results differ, the present experiment was undertaken, in part, as an attempt to resolve this conflict.

Reprint requests should be sent to Zita E. Tyer, Department of Psychology, George Mason University, 4400 University Drive, Fairfax, Virginia 22030.
Only Coltheart (1969) has tested the effect of verbal distance information on observers' size estimates. In Coltheart's (1969) experiment, subjects judged the size of a stimulus more accurately when they were told its distance than when they were not. It is unfortunate, however, in terms of stimulus control, that the instruction was the same distance as that at which the object was actually located. It is not possible to determine if the accurate judgments were entirely a function of the instruction or were enhanced by uncontrolled distance cues.

Since the purpose of this experiment was to test the effect of both size and distance instruction on distance and size estimates when other cues were demonstrably absent, stimulus distances and retinal images were chosen so that comparisons within the experiment would permit tests of the effectiveness of cue reduction.

\section{METHOD}

\section{Subjects}

The subjects in this experiment were 160 experimentally naive volunteers from psychology courses at George Mason University. All subjects had at least $20 / 30$ vision in one eye, as measured with a Snellen eye chart.

\section{Apparatus}

The experimental area consisted of three adjoining interior rooms and part of a common hallway $(8.02 \times 60.3 \mathrm{ft})$ which was secured and made light-tight. This laboratory suite was internal to other laboratory rooms and separated by them from the exterior hallway, so that subjects could gain no idea of the shape or extent of the experimental tunnel.

The subjects were seated on an adjustable metal stool immediately behind a head- and chinrest (Narco Biosystems, Inc.).

Stimuli were constructed from $6.09 \times .82$ in. electroluminescent metal strips (John Meshna, Inc., Lynn, Mass.). When an alternating current was applied to a strip, it emitted a uniform faint green glow across its width. Masking the strips with black electrical tape produced four stimuli with the following dimensions-2.0 $\times$ $.43 \mathrm{in} .(5.08 \times 1.11 \mathrm{~cm}), 4.0 \times .87$ in. $(10.16 \times 2.22 \mathrm{~cm}), 1.0 \times .22 \mathrm{in}$. 
$(2.54 \times .56 \mathrm{~cm})$, and $2.0 \times .43 \mathrm{in} .(5.08 \times 1.11 \mathrm{~cm})$. The strips were mounted on wooden stands $(3.5 \mathrm{ft}$ high) perpendicular to a subject's eye. One stimulus of $2.0 \mathrm{in}$. was placed $12 \mathrm{ft}$ from the subject's eye and subtended a visual angle of $48 \mathrm{~min}$; the other was placed at $48 \mathrm{ft}$ and subtended $12 \mathrm{~min}$. The stimuli of 4.0 and 1.0 in., both placed at $24 \mathrm{ft}$, subtended 48 and $12 \mathrm{~min}$, respectively.

Prior to the experiment, brightness matches between the stimuli were empirically determined by adjusting supply voltages to the strips with ac variacs. Luminance was $-.80 \log \mathrm{fL}$, as measured at the position of the eye with an SEI photometer. The unusually low stimulus luminances, the absence of any other light source in the light-sealed experimental area, the unfamiliar dimensions of this area, and monocular viewing without head motion were designed to eliminate all cues or "anchor effects" except accommodation or (monocular) accommodative convergence.

\section{Design}

The subjects were assigned randomly to one condition of a $4 \times 4$ factorial design. The two independent variables were four information conditions (NIS, NID, IS, ID) and four stimulus conditions (A, B, C, D).

The first independent variable consisted of the presence or absence of size or distance information. The NI (no information) conditions served as control conditions. Subjects in these groups were simply required to estimate either the size (NIS) or the distance (NID) of the object. The remaining two groups were experimental groups. Subjects in the ID group were told that the size of the object was $2 \mathrm{in}$. from top to bottom and were asked to estimate its distance. Subjects in the IS group were told that the distance of the object was $24 \mathrm{ft}$ and were asked to estimate its size from top to bottom.

The second independent variable was stimulus condition. The four stimulus conditions were designed to produce two identical visual angles using different sizes and distances.

\section{Procedure}

The subject, after being tested for acuity and receiving instructions, was blindfolded, led into the experimental area, seated, and had the head-and chinrest adjusted. He was asked to remove one of the eyepatches, and then, depending on his treatment condition, was told to estimate either the size or the distance of the object. At this time, subjects in the ID and IS groups were given the additional information that the object was 2 in. in height or $24 \mathrm{ft}$ away, respectively. Total time, from blindfolding until an estimate was given, was approximately $2 \mathrm{~min}$.
The room lights were then turned on and full-cue judgments were taken. A subject who had estimated size was asked to draw on a piece of paper a line whose length was equivalent to the estimate. An unmarked straight-edge was provided for this purpose. Subjects who estimated distance were asked to remain seated and to estimate the distance of the stimulus stands (which were 12, 24, and $48 \mathrm{ft}$ in front of them) in feet. These estimates were used in a regression equation yielding a calibration score which was each subject's predicted estimate of an object at $24 \mathrm{ft}$ based on fullcue estimates. Calibration estimates were taken because, as Gogel and Tietz (1973) suggest, they depict more accurately what the subjects really mean by their cue-reduced estimates.

\section{RESULTS}

\section{Judgment of Size}

A $2 \times 4$ analysis of variance for uncorrelated factors was carried out for the verbal size estimates. In this analysis, stimulus differences could be partitioned into those due to the retinal image size of the stimulus $(\mathrm{A}+\mathrm{B}$ vs. $\mathrm{C}+\mathrm{D})$ and those due to other stimulus characteristics (see Table 1). In this case, "other stimulus characteristics" would be differences in metric size and/or distance, which should be undetectable if cue reduction was successful.

The analysis of variance revealed that differences due to instruction and retinal image size were significant (see Table 1). Inasmuch as other stimulus characteristics had no effect, it appears that cue reduction was successful. There was no interaction between instructions and any stimulus characteristic.

An entirely parallel analysis of variance was carried out for the perceptual-motor size estimates. It is also shown in Table 1 and yielded quite similar results.

These analyses show that for both verbal and perceptual-motor motor measures, the large retinal equalities $(A+B)$ yielded significantly larger size judgments than the small retinal equalities $(C+D)$ for both verbal and perceptual-motor judgments.

Table 1

ANOVA for Verbal and Perceptual-Motor Size Estimates

\begin{tabular}{|c|c|c|c|c|}
\hline Source & df & MS & $\mathbf{F}$ & $\mathrm{p}$ \\
\hline \multicolumn{5}{|c|}{ Verbal Size Estimates } \\
\hline Instructions (I) & 1 & 127.59 & 16.34 & $<.01$ \\
\hline Stimulus Differences (S) & (3) & & & \\
\hline Retinal Angle Differences (R) & 1 & 46.31 & 5.92 & $<.05$ \\
\hline Other Stimulus Differences (O) & 2 & 15.33 & 1.96 & \\
\hline$I \times S$ & (3) & & & \\
\hline I $\times \mathrm{R}$ & 1 & 2.12 & .27 & \\
\hline$I \times O$ & 2 & 16.21 & 2.08 & \\
\hline Error & 72 & 7.81 & & \\
\hline \multicolumn{5}{|c|}{ Perceptual-Motor Size Estimates } \\
\hline Instructions (I) & 1 & 81.87 & 11.43 & $<.01$ \\
\hline Stimulus Differences (S) & (3) & & & \\
\hline Retinal Angle Differences (R) & 1 & 36.54 & 5.10 & $<.05$ \\
\hline Other Stimulus Differences (O) & 2 & 8.23 & 1.15 & \\
\hline$I \times S$ & (3) & & & \\
\hline$I \times R$ & 1 & 3.11 & .43 & \\
\hline$I \times 0$ & 2 & 13.10 & 1.83 & \\
\hline Error & 72 & 7.16 & & \\
\hline
\end{tabular}


Newman-Keuls analyses showed that $A$ did not differ from B, under the NIS condition, and that $C$ did not differ from $D$. Thus, judged size corresponded to retinal, not metric, size. However, a 4-to-1 ratio of retinal size produced only a 2.5 -to-1 ratio in verbal judgments of size, or a 2.7-to-1 ratio in perceptualmotor judgments (see Table 2).

Both analyses also show that when subjects were given a distance instruction, their size judgments changed in a reliable manner in a direction appropriate to the distance instruction. Inspection of the means for the IS condition (see Table 2) reveals that instruction had relatively less effect on judgments in stimulus condition $\mathbf{A}$. Thus, while $\mathbf{A}$ was equivalent to $B$ in the NIS condition, a Newman-Keuls analysis shows that it is not in the IS condition. With instruction, verbal size judgments for B are significantly larger than for any of the other stimuli $(p<.05)$, which are all equivalent. That is, $B>A=C=D$. Although the perceptual-motor judgments show the expected ordinal effect a little better than the verbal judgments, the Newman-Keuls analysis shows a similar result; again, $\mathrm{B}>\mathrm{A}=\mathrm{C}=\mathrm{D}$ with $\mathrm{p}<.05$ when $\mathrm{B}$ is compared with any other stimulus. Thus, judgments for $A$ were more like those for $C$ and $D$ than B, even for the perceptual-motor judgments. This irregularity should not be attributed to the size or distance of A, given the NIS results. Instead, it probably reflects subjects' great difficulties in making sensible size estimates even with instructions (Gogel \& Tietz, 1973).

In sum, instruction had a significant effect, and with instruction condition B produced a significantly greater mean than the other conditions. Yet, there was no significant interaction between stimulus condition and instruction. Hence, the fairest interpretation is that instruction had the general effect of increasing size judgment for all stimuli and produced its largest effect on the B condition.

A comparison of the size judgments actually obtained for the IS condition with those that the sizedistance invariance hypothesis would actually yield for the large and small retinal images with that instruction yielded mixed results. For both the verbal

Table 2

Means in Inches for Verbal and Perceptual-Motor Size Estimates

\begin{tabular}{|c|c|c|c|c|}
\hline \multirow[b]{2}{*}{ Condition } & \multicolumn{2}{|c|}{ Verbal } & \multicolumn{2}{|c|}{ Perceptual-Motor } \\
\hline & NIS & is & NIS & IS \\
\hline A & 1.96 & 3.15 & 1.68 & 2.51 \\
\hline B & 1.85 & 6.43 & 1.39 & 5.45 \\
\hline C & .63 & 2.24 & .54 & 2.23 \\
\hline D & .86 & 3.65 & .67 & 2.24 \\
\hline \multicolumn{5}{|c|}{ Collapsed for Identical Retinal Angles } \\
\hline $\begin{array}{l}A+B \\
C+D\end{array}$ & $\begin{array}{r}1.90 \\
.71\end{array}$ & $\begin{array}{l}4.79 \\
2.94\end{array}$ & $\begin{array}{r}1.54 \\
.58\end{array}$ & $\begin{array}{l}3.98 \\
2.24\end{array}$ \\
\hline
\end{tabular}

and perceptual-motor size estimates, analysis of $\mathbf{A}+$ $B$ revealed no significant difference from the predicted value of 4 in. $[t(18)=.87, p>.05, t(18)=.94$, $p>.05$, respectively]. However, both types of estimates obtained for $C+D$ differed significantly from the predicted value of 1 in. $[t(18)=2.22, p<.02$, $t(18)=2.93, p<.005$, respectively].

The difference in the $t$ values obtained for the large and small retinal images partially reflects the greater variability obtained with the large retinal images. However, a comparison of the pooled scores obtained with the large and small images (see Table 2) suggests that subjects greatly overestimated the true size of the small retinal images in the face of instruction, but produced relatively accurate estimates for the large retinal images.

\section{Judgment of Distance}

Since the verbal distance estimates contained extreme scores, they were ranked, and Kruskal-Wallis analyses of variance for ranks were calculated. For comparison purposes, a Kruskal-Wallis analysis was also calculated for the collapsed conditions contrasting the data for the two retinal equalities as a function of the NID-ID condition.

Parallel analyses were carried out for the calibrated distance estimates.

The analysis of variance of ranks showed no significant differences for the verbal and calibration distance estimates with no instruction (NID) [H(3)= $.837, \mathrm{p}>.05$, and $\mathrm{H}(3)=2.48, \mathrm{p}>.05$, respectively]. It appears that uninstructed distance judgments represent only a normal dispersion of responses, ${ }^{2}$ and do not reflect the true size or distance of the stimuli or their retinal images. The fact that the uninstructed distance judgments do not reflect the true distances of the stimuli supports the inference drawn from the size judgments that there were no effective uncontrolled cues to distance (see Table 3).

Since the estimates of distance did not vary significantly in the absence of instruction, indicating that there were no effective cues to physical size and distance, subsequent analysis was based on the remaining variable, visual angle, in combination with instruction. Thus, upon being told that a stimulus is 2 in., a subject viewing a large retinal image should judge it as relatively near; in contrast, a subject receiving the same instruction but viewing a small retinal image should judge it as relatively far. The appropriate constrast to show this instruction effect is to pool the judgments for the large retinal image (A and $B$ ), to pool those for the small retinal image (C and D), and to compare these when subjects did or did not receive the size instruction. This comparison, which is shown in Table 3, revealed that judgments changed in the appropriate directions, and that the change was strong enough to yield a significant effect $[H(3)=8.78, p<.025$, and $H(3)=8.42, p<.025$, 
Table 3

Medians in Feet for Verbal and Calibration Distance Estimates

\begin{tabular}{|c|c|c|c|c|}
\hline \multirow[b]{2}{*}{ Condition } & \multicolumn{2}{|c|}{ Verbal } & \multicolumn{2}{|c|}{ Calibration } \\
\hline & NID & ID & NID & ID \\
\hline $\mathbf{A}$ & 10.00 & 8.00 & 11.59 & 10.08 \\
\hline B & 12.00 & 8.00 & 14.64 & 10.09 \\
\hline C & 8.00 & 10.00 & 11.21 & 15.75 \\
\hline D & 8.00 & 13.5 & 9.31 & 15.61 \\
\hline \multicolumn{5}{|c|}{ Collapsed for Identical Retinal Angles } \\
\hline $\begin{array}{l}A+B \\
C+D\end{array}$ & $\begin{array}{r}10.00 \\
8.00\end{array}$ & $\begin{array}{r}8.00 \\
11.00\end{array}$ & $\begin{array}{l}13.13 \\
10.12\end{array}$ & $\begin{array}{l}11.69 \\
14.55\end{array}$ \\
\hline
\end{tabular}

with directional tests for the verbal and calibrated estimates, respectively]. While all individual comparisons are in the right direction, none are sufficiently robust to be statistically significant.

Note that the effect is clearly an interaction of instruction with retinal image, since, overall, the ID judgments are not significantly larger or smaller than the NID judgments, whether verbal $[\mathrm{U}(40,40)=783$, $\mathrm{p}>.05]$ or calibrated $[\mathrm{U}(40,40)=707, \mathrm{p}>.05]$. Thus, the size instruction did not introduce any constant error but, rather, produced an effect that varied predictably with retinal image size.

\section{DISCUSSION}

The demonstration that information regarding object size affects judgments of an object's distance is congruent with similar results obtained by Baird (1963) and Gogel (1981). Unlike previous research, however, the present study incorporated specific tests to assess the possible effects of uncontrolled distance cues. It also conveyed size information, not by verbally equating stimulus size with the familiar size of some object, but by specifying size in metric units. This procedure is similar to that of Coltheart (1970) and Park and Michaelson (1974).

The overall effect of the size instruction was to produce a small change in distance judgments. Without instructions, distance judgments were independent of retinal image size, and were normally distributed around $9.5 \mathrm{ft}$. This result is congruent with Baird's (1964) findings that perception of retinal image size is not correlated with the perception of stimulus distance in a cue-reduced situation. With instructions, however (i.e., that the object was 2 in. tall), the smaller images tended to be judged as farther away (i.e., around $11.0 \mathrm{ft}$ ) in contrast to large images, whose apparent distance was $8.0 \mathrm{ft}$. It should be noted that these effects represent only a fraction of those predicted by the size-distance invariance hypothesis (SDIH). The limited magnitude of the effect may be explained by considering the size judgments that subjects made when not given size instructions. That is, larger retinal images were believed to be only slightly less than 2 in.; therefore, instructions that an object was 2 in. should not and did not have a strong effect on the judged distance of the large stimuli. However, the stimuli with small retinal images were believed to be less than $1 \mathrm{in}$. in the absence of instructions, and the 2 -in. instruction could and did have a stronger effect on judgments of their distance. This effect was restricted, however, even for the small stimuli, by the fact that verbal reports tend to understate greatly either real or simulated large distances (Gogel, 1968, 1969a, 1969b, 1976). This also reflects a limitation on the use of the SDIH as a mathematical expression for predicting effects that are not purely perceptual (Gogel \& Sturm, 1971).

In Park and Michaelson's (1974) experiment, the effect of size instructions was nonsignificant. It is possible that accommodation or monocular accommodative convergence attenuated the effect of their instructions, since the stimulus distance was only $6 \mathrm{ft}$, although other methods of conferring a known (familiar) size on the object were successful. It may also be that the size instructions, 1.5 or 3 in. (veridical), were not effective because they did not differ enough from the subject's uninstructed size perception. There is no way to determine the latter.

Coltheart's (1970) size instructions produced distance judgments much closer to those predicted by the SDIH than were those found in the present experiment. The difference may reside in the fact that the distance predicted from Coltheart's veridical instruction was not different from what would have been indicated by accommodation or monocular accommodative convergence; the distance predicted from the other instruction coincides with that predicted from the specific distance tendency (SDT) later discovered by Gogel and Tietz (1973). It is possible, therefore, that these factors reinforced the effect of size instructions in Coltheart's experiment. Other differences between the experiments are that Coltheart's stimuli were larger, generally nearer $(4.12 \mathrm{~m})$, and shed more illumination. Again, it is impossible to determine what size or distance perceptions Coltheart's subjects had prior to instructions.

It should be noted that the median distance judgment $(9.5 \mathrm{ft})$ obtained in the present experiment in the absence of instructions is not a bad fit for the 2$3 \mathrm{~m}$ predicted by the SDT. Since this does not coincide with the 24- $\mathrm{ft}$ distance instruction, the latter must have had its effect in spite of the SDT. Likewise, the changes in distance judgments occasioned by the size instruction occurred in spite of any anchoring effect the SDT may have had. Thus, while the SDT may mitigate the effects of instructions, it does not prevent them. Similarly, Coltheart's (1970) size instructions had an effect in spite of the SDT when they were incompatible with it.

In assessing the effect of the distance instruction on judged size, it is important to note that subjects were able to judge size differentially for the different 
retinal images, even in the absence of distance information. These results are in accord with those of Gogel (1969c) and Gogel and Sturm (1971) as well as those of Baird (1964). The general pattern of results was that sizes were underestimated, especially for small stimulus objects. This, of course, would be predicted from the SDIH, since the distances were generally underestimated in the absence of instructions. However, increasing distance information via instructions should increase size judgments. Size judgments actually did increase (more than would be predicted for the case of the small stimuli) when distance information was given. While the resulting judged sizes for the larger stimuli were relatively closer to the physical size that would produce the given retinal angle at the instructed distance, judged sizes of the smaller retinal images were unexpectedly exaggerated. Thus, while distance instructions affected size judgments in the expected direction, the effect was not precise. Still, perceptual-motor judgments of size were not greatly different from the actual sizes of 4 and 1 in. This is particularly interesting in view of the fact that the distance instruction (information) was $24 \mathrm{ft}$. Subjects are probably not very familiar with this large a distance, as compared with an inch, a foot, a yard, or $6 \mathrm{ft}$, which have everyday referents.

Only Coltheart's (1969) experiment provides an alternative assessment of the effect of distance instructions on size judgments. For the one retinal size, metric size, and distance tested, the veridical instruction produced a good fit to predictions from the SDIH. The judgments obtained in the absence of a distance instruction are easily explained in terms of the SDT. Coltheart's experiment may be interpreted similarly to the present one; judgments are compatible with the SDT unless instruction is opposed.

Since the issues involved in size and distance perception are complex, the present data must be interpreted conservatively and empirically. There were no observable effects of uncontrolled cues, and both size and distance information had identifiable effects in spite of any effect the SDT might have had. In the absence of instruction, retinal images of different sizes could be differentiated, but stimulus sizes were underestimated; stimulus distances could not be differentiated, but coincided with those predicted from the SDT. Distance instructions increased the judged sizes of the stimuli. Size instructions also had modest effects, in the directions predicted by the SDIH.

\section{REFERENCES}

BAIRD, J. C. Retinal and assumed size cues as determinants of size and distance perception. Journal of Experimental Psychol$o g y, 1963,66,155-162$.

Baird, J. C. Size of retinal image as a perceptual cue. Perceptual and Motor Skills, 1964, 18, 529-532.

Coltheart, M. Effects of two kinds of distance information on visual judgments of absolute size. Nature, 1969, 221, 388.

Coltheart, $M$. The effect of verbal size information upon visual judgments of absolute distance. Perception \& Psychophysics, $1970,9,222-223$.

Fitzpatrick, V., Pasnak, R., \& Tyer, Z. E. The effect of familiar size at familiar distances. Perception, 1982, 11, 85-91.

Gogel, W. C. The effect of set on perceived egocentric distance. Acta Psychologia, 1968, 28, 283-292.

GoGEL, W. C. The absolute and relative size cues to distance. American Journal of Psychology, 1969, 82, 228-234. (a)

Goael, W. C. The effect of object familiarity on the perception of size and distance. Quarterly Journal of Experimental Psychology, 1969, 21, 239-247. (b)

Goges, W. C. The sensing of retinal size. Vision Research, 1969, 9, 1079-1094. (c)

GoGEl, W. C. An indirect method of measuring perceived distance from familiar size. Perception \& Psychophysics, 1976, 20, 419-429.

GoasL, W. C. The role of suggested size in distance responses. Perception \& Psychophysics, 1981, 30, 149-155.

Goges, W. C., \& Mentens, H. W. Perceived size and distance of familiar objects. Perceptual and Motor Skills, 1967, 25, 213-225.

Gogen, W. C., \& Sturm, R. D. Directional separation and the size cue to distance. Psychologische Forschung, 1971, 35, 57-80.

Gooe L, W. C., \& Tretz, J. D. Absolute motion parallax and the specific distance tendency. Perception \& Psychophysics, 1973, 13, 284-292.

Park, J. N., \& Michaelson, G. J. Distance judgments under different size-information conditions. Perception \& Psychophysics, 1974, 15, 57-60.

\section{NOTE}

1. A chi-square goodness of fit test indicates no significant departure from normality $\left[\chi^{2}(3)=.76, p>.05\right]$. There is some suggestion of a rough positive correspondence between the judged distances and judged sizes of the stimuli in the absence of instructions, but it is not statistically significant; it should be remembered that size and distance judgments were made by different subjects. Gogel and Sturm (1971) have suggested that such relation would reflect the operation of a size-distance invariance principle in a purely perceptual situation in which the subject has no "cognitive" size information or impressions.

(Manuscript received June 15, 1982; revision accepted for publication March 16, 1983.) 